
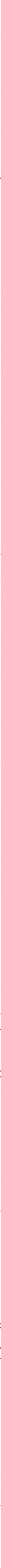
Refinement of the analysis and confirmation of the results continues. We are currently drafting 3 papers on high-redshift, $\mathrm{X}$-ray selected and optically-selected subsets of the sample along with the main database paper to present all our results. Dr. Kuraszkiewicz joined us to work on the X-ray sample paper but in the spring both Dr. Hooper and Dr. Kuraszkiewicz left the ISO work to take up new positions. This has hampered the completion of our various papers, though they still move forward at a slower pace.

Dr. Hooper spent much of his time working on the final processing of the ISO data as well as using the ISO and other published data on high redshift AGN to make estimates of the star formation rate at high redshift and thus concluding that much of the far-IR emission from these high-redshift AGN is likely to be powered by the AGN rather than star formation. This work is being written up as part of the paper on the high redshift ISO sources.

The paper reporting the ISO results on hard X-ray selected AGN is close to completion. We find that, as predicted, the IR continuum of these sources extends to redder sources than in optically/radio selected sample. This indicates that the latter samples miss a portion of the population which is fainter in the optical but can be easily picked up in the hard X-ray.

We started discussions with Dr. Wood, now at St. Andrews, on modelling the full IR continuum using his dusty disk models. This has progressed well with the models fitting the data pretty well, though rather large disks are required to fit the cooler, long wavelength emission. From the fits we can obtain estimates of the mass and inclination of the system. We are currently considering whether to include these detailed fits in another papers or in one of the existing ones.

\section{Talks and Posters:}

Dr. Hooper presented a talk at the 197th AAS meeting: "SEDs and Star-Formation Rates in High-redshift AGN", Jan 2000

Dr. Hooper presented a poster at "Advanced Lectures on the Starburst-AGN Connection," INAOE, Mexico, 2000

Dr. Hooper presented a talk at the 6th Annual Stromlo AGN Workshop, MSO, Canberra, Australia, 2001.

Visits:

Dr. Wilkes visited Dr. Wood in St. Andrews, Scotland in July 2001.

Abstracts and Papers:

SEDs and Star-Formation Rates in High-redshift AGN (E.J. Hooper, B.J. Wilkes, K.K. McLeod, M.S. Elvis, D.H. Hughes, C.D. Impey, J.K. Kuraszkiewicz, C.J. Lonsdale, M.A. Malkan \& J.C. McDowell), BAAS, 32, 1436, 2000

The Far-infrared Spectral Energy Distributions of Quasars, (Wilkes, B.J.), invited review in "The Far-Infrared and Submillimeter Spectral Energy Distributions of Active and Starburst Galaxies", eds. I. Van Bemmel, Peter Barthel, Belinda Wilkes, New Astronomy Reviews, in press (copy attached to this report) 


\title{
The far-infrared spectral energy distributions of quasars
}

\author{
Belinda J. Wilkes, ${ }^{\text {a }}$

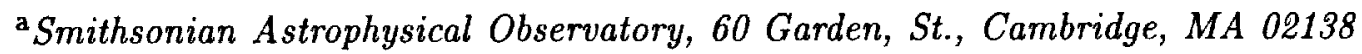

\begin{abstract}
The origin of the infrared emission in Active Galactic Nuclei (AGN), whose strength is comparable to the optical/ultra-violet (OUV) emission, is generally thought to be a combination of thermal emission from dust and non-thermal, synchrotron emission. Although data are sparse, particularly in the far-infrared, the broad wavelength range of this emission suggests a wide range of temperatures and a combination of AGN and starburst heating mechanisms. The strength of the non-thermal emission is expected to be related to the radio emission. While this scenario is wellestablished, basic questions, such as the spatial and temperature distribution of the dust, the relative importance of AGN and starburst heating and the significance of the non-thermal contribution remain largely undetermined.

The wide wavelength range of the Infrared Space Observatory (ISO) combined with its arcmin spatial resolution and increased sensitivity facilitated the observation of a larger subset of the AGN population than previously covered, allowing these questions to be investigated in more detail. This paper will review the spectral energy distributions (SED) of AGN with particular emphasis on the infrared emission and on ISO's contributions to our knowledge. Preliminary results from ISO observations of X-ray selected and high-redshift AGN will be described.
\end{abstract}

Key words: Active Galactic Nuclei, spectral energy distributions

\section{Introduction}

The standard model for an AGN includes a super-massive black hole (SMBH) surrounded by an accretion disk in the center of a galaxy. The central regions of the AGN produce strong hard X-ray emission (and relativistic jets in radioloud sources) along with thermal emission from the accretion disk. Much of this radiation is re-processed by dust and gas within the galaxy, modifying the optical-X-ray spectral energy distribution (SED) and resulting in strong 
infrared emission from hot material further out in the galaxy such as the putative opaque dusty torus surrounding the accretion disk which is dictated by AGN unification schemes. Thus the appearance of the SED depends upon factors such as the amount and nature of obscuring material, the line of sight at which we observe each source and the amount of beamed emission related to the jets. In order to understand the AGN energetics and physics, we need to be able to disentangle these effects and probe the fundamental origins of the emission. This requires as a first step making full, multi-wavelength observations to determine the SED of a sample of AGN unbiased by the effects listed above.

Existing measurements of the infrared continuum of AGN remain sparse. IRAS observed small subsets of the infrared-brightest AGN drawn from a variety of different samples and so its ground-breaking results are severely limited in their application to the full population. By contrast our observational knowledge of the AGN SED over the remainder of the radio-X-ray energy bands is good (Sanders et al. 1989, Elvis et al. 1994). The infrared-UV SED of AGN are generally independent of their type or radio-loudness: differences between radio-loud and radio-quiet exist primarily in their X-ray spectra and strength. IRAS and submm measurements of the IR-brightest AGN show a farinfrared (FIR) spectrum which is too steep to be consistent with non-thermal, synchrotron emission below the self-absorption frequency $(\alpha>2.5)$. Indeed known synchrotron sources generally consist of several inhomogeneous components and so have observed far-infrared slopes $\sim 1$ (Gear et al. 1994). Thus it was concluded that the infrared emission of radio-quiet AGNs and lobedominated, radio-loud AGNs is dominated by thermal emission from cool and warm dust (Chini et al. 1989, Barvainis 1990, Hughes et al. 1993, Antonucci et al. 1990). Since the AGN generally have warm infrared colors compared with galaxies, it is clear that at least some of the dust must be heated by the central AGN (Barvainis 1990). By contrast, core-dominated, radio-loud AGNs show a continuous IR-radio SED. These sources have emission components which are beamed towards us and are thought to have an additional, non-thermal infrared component linked to this radio emission (Courvoisier 1998, Tuerler et al. 1999, Hoekstra et al. 1997).

While this scenario is generally successful, it is based upon a few, relatively IR-bright AGN of each type so that its application to the general population remains questionable. In addition, several observational results call it into question. For example, near-infrared variability studies show correlated variations with magnitudes similar to those observed in the optical out to wavelengths as long as $10 \mu \mathrm{m}$. Given the estimated size ( $\sim 20 \mathrm{lyr}$; Neugebauer \& Matthews 1999) of the $10 \mu \mathrm{m}$ dust emitting region, these variations are difficult to explain as dust reverberation and suggest a significant contribution from non-thermal emission in these few sources. There also appears to be 

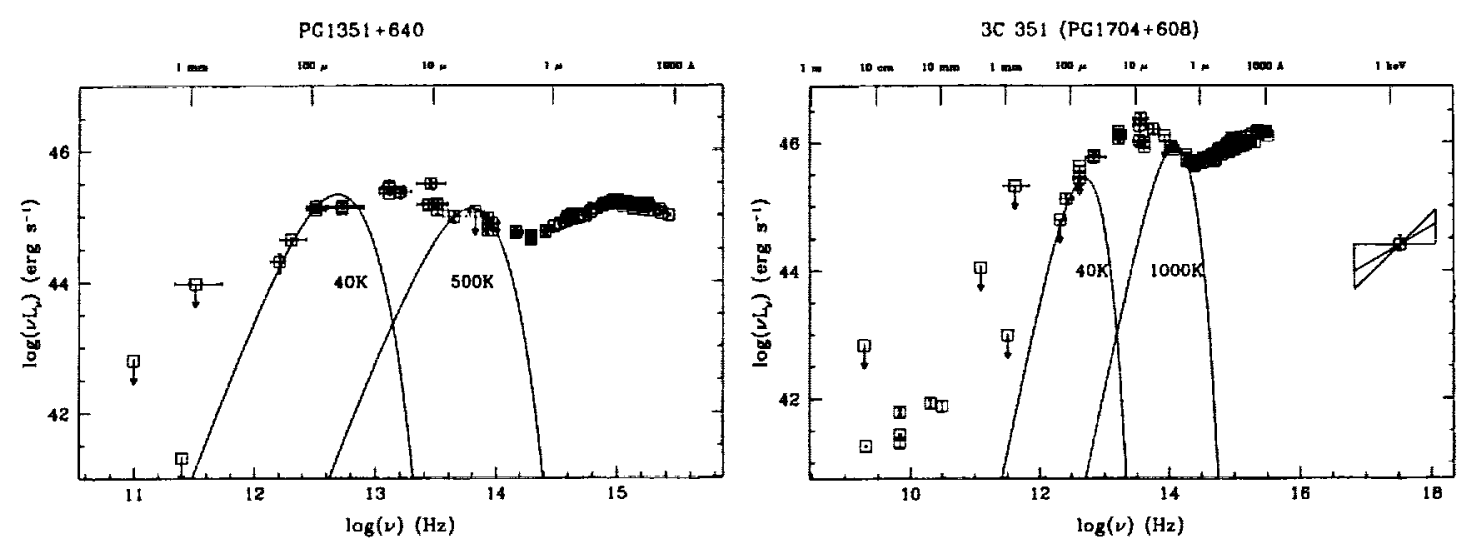

Fig. 1. Radio-UV SED of two low-redshift AGN observed as part of our ISO sample: (left) a radio-quiet source, PG 1351+640; (right) a lobe-dominated, radio-loud source, $3 \mathrm{C} 351$, illustrating the broad, smooth infrared continuum and steep far-infrared cut-off typical of these sources. Grey-body curves assuming $\beta=2$ are super-posed indicating the range of temperature present.

a preferential near-infrared/X-ray relation (Brissenden 1989, Carleton et al. 1987, Green et al. 1992) which implies related emission mechanisms for the nuclear fluxes, although interpretation in terms of dust heated by the X-ray continuum is also a possibility (Krabbe et al. 2000).

We use $\mathrm{H}_{0}=75 \mathrm{~km} \mathrm{~s}^{-1} \mathrm{Mpc}^{-1}$ and $\mathrm{q}_{0}=0.5$ throughout this paper.

\section{ISO's contribution.}

Several ISO programs aimed to expand the sample of far-infrared observed AGN. Although these samples remain confined to those which are relatively IR-bright, significant progress has been made. The ISO results (Hooper et al. 1999a,b, Wilkes et al. 1999, 2000, Haas et al. 1998, 2000) generally confirm and strengthen the earlier thermal-dominated infrared scenario with broad, smooth infrared continua and steep far-infrared cut-offs when these could be observed (Figure 1). This reinforces current models in which a moderately thick, extended dusty disk (Granato \& Danese 1994, Granato et al. 1997, Efstathiou \& Rowan-Robinson 1995) generates the broad near-mid-infrared continuum, with the cooler, far-infrared emission generated further out in the galaxy, probably by a starburst. Earlier models of an optically thick, compact torus (Pier \& Krolik 1992) generate emission over too narrow a range of temperatures to match the broad, observed continua.

A decrease in strength of the non-thermal component and resulting increased dominance of the warm dust emission is observed as the AGN decreases in radio-loudness (Haas et al. 1998, Polletta et al. 2000 and in these proceedings). Decomposition of the FIR SED in Seyfert galaxies and quasars has demonstrated the presence of cool and warm dust components (Figure 2 of Wilkes et al. 1999, see also Pérez García et al. 1998, Polletta et al. 2000), quan- 

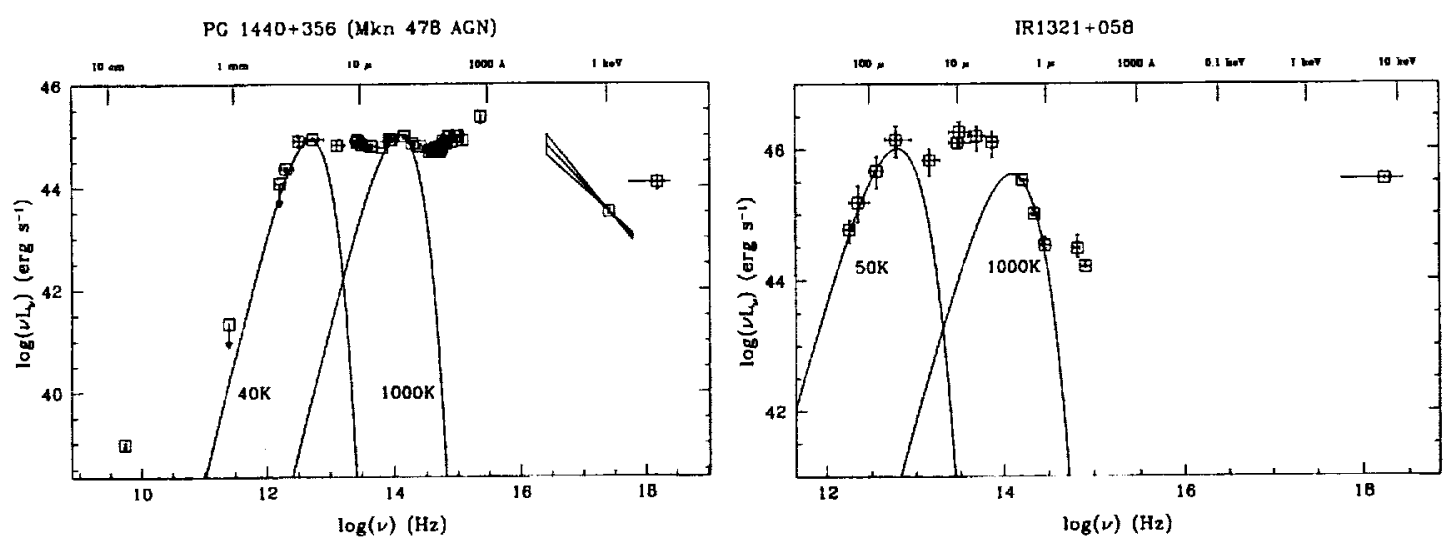

Fig. 2. Far-infrared-X-ray SED of the X-ray selected AGN with the (left) bluest (PG 1440+356) and (right) reddest (IRAS 1321+058) near-infrared continuum illustrating the wide range of infrared continuum shapes present in the $\mathrm{X}$-ray selected sample. Grey-body curves assuming $\beta=2$ are super-posed indicating the range of temperature present.

tified their absolute and relative strengths and begun to probe their spatial distributions (Tansley et al. 2001). Comparison with ISO observations of radio galaxies (Van Bemmel et al. 2000 and in these proceedings) suggests that the warm dust remains optically thick to fairly long wavelengths $(90-160 \mu \mathrm{m})$.

\section{X-ray selected AGN}

Hard X-ray selection is arguably the optimal method for defining a representative sample of AGN. Hard X-rays are relatively unbiased by the affects of obscuration and re-processing along the line-of-sight intrinsic/external to the AGN which result in unknown fractions of the population being missed from traditional optical/soft-X-ray samples. Our ISO sample included 21 hard Xray selected AGN from the HEAO A-1 Source Catalog (Wood et al. 1984, Remillard et al. 1986), 12 Seyfert 1's, 1 Seyfert 2 and 7 intermediate AGN and 1 unclassified. With the exception of one border-line source, KUV $1821+64$, all are radio-quiet. They were observed with ISOPHOT at 8 wavelengths: $5,7,12$, $25,60,100,130,200 \mu \mathrm{m}$, in either/both rectangular chopping mode or small rasters. We combined ISO and multi-wavelength data to form far-infrared-Xray $\mathrm{SED}$.

\subsection{Infrared continuum characteristics}

As is typical of AGN, the infrared continua of the HEAO sources is broad, comparable in strength to the optical/UV emission and often shows a minimum at $\sim 1 \mu \mathrm{m}$. The SED show a wide distribution in IR continuum shapes. This is illustrated in Figure 2 which shows the SED of two AGN with extremely 
Table 1

Infrared continuum characteristics of the X-ray selected AGN

\begin{tabular}{|c|c|c|c|c|c|c|c|}
\hline Name & $z$ & $\begin{array}{l}\text { ISO } \\
\text { Date }\end{array}$ & $\begin{array}{l}L_{3-60}^{1} \\
\operatorname{erg~s}^{-1}\end{array}$ & $\begin{array}{l}L_{60-100}^{1} \\
\mathrm{erg} \mathrm{s}^{-1}\end{array}$ & $25 / 60^{2}$ & $\mathrm{X} / 100^{2}$ & $\begin{array}{l}L_{\text {bol }}^{1} \\
\operatorname{erg~s}^{-1}\end{array}$ \\
\hline \multirow[t]{2}{*}{ H0557-385 } & 0.0344 & $1 / 22 / 1998$ & 45.16 & 43.47 & 1.20 & 0.86 & 45.31 \\
\hline & & $11 / 7 / 1997$ & & & & & \\
\hline \multirow[t]{2}{*}{ MCG -6-30-15 } & 0.008 & $7 / 30 / 1996$ & 43.83 & 43.05 & 0.57 & 0.15 & 44.29 \\
\hline & & $2 / 17 / 1996$ & & & & & \\
\hline IRAS $1321+058$ & 0.205 & $7 / 9 / 1996$ & 46.55 & 45.58 & 0.26 & 0.63 & 46.74 \\
\hline PG $1440+356$ & 0.079 & $6 / 2 / 1996$ & 45.34 & 44.79 & 0.28 & -0.16 & 45.98 \\
\hline MR 2251-178 & 0.066 & $5 / 22 / 1996$ & 45.12 & 44.4 & 0.40 & 0.52 & 45.88 \\
\hline
\end{tabular}

red and blue infrared continua respectively. Table 1 lists various broad-band continuum luminosities and ratios along with an estimate of the bolometric luminosity for several, representative X-ray selected AGN from our sample. The temperatures of the dust are not well-constrained by the data, even if optically thin emission is assumed. Estimates based upon comparison with grey-body curves indicate a typical range of dust temperatures of $\sim 50-500 \mathrm{~K}$.

As discussed above, the clearest way to confirm thermal emission is by observing a far-infrared cut-off slope $>2.5$, the steepest possible for non-thermal, synchrotron emission. There is only one case where a clear far-infrared turnover is observed: MR 2251-178, with a far-infrared slope of 3.7 (Figure 3). In all other cases the far-infrared slopes are $<2.5$ which implies, in a thermal scenario, the presence of a longer wavelength, cooler dust component. Although the lack of a steep turnover does not allow us to rule out non-thermal emission in these sources.

\subsection{Comparison with optically/radio-selected AGN}

We compared the infrared continuum and full SED of our hard X-ray selected sample with the compilation of SED for low-redshift, radio and optically selected AGN (Elvis et al. 1994). Despite their different selection the soft X-ray and optical luminosities of these two samples are very similar though the latter extends to higher redshift. The primary difference between the SED of the two samples is illustrated in Figure 4 which shows an extension to redder optical colors in the X-ray selected sample. This is expected due to the reduced bias against such sources when selecting in the hard X-ray band. A Kolmogorov- 


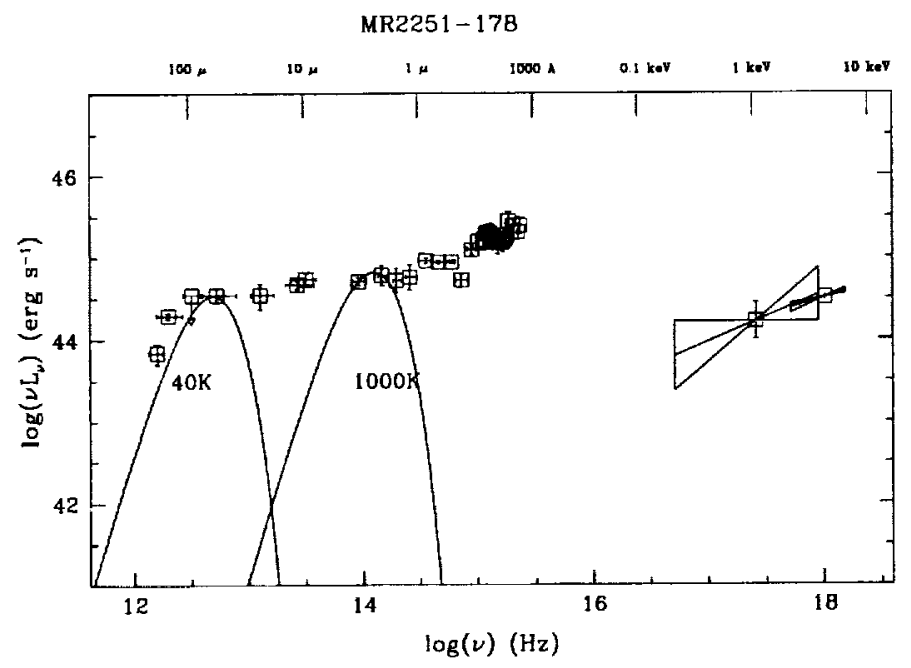

Fig. 3. The far-infrared-X-ray SED of MR $2251-178$, the only source for which a clear, thermal far-infrared turnover is observed. The figure shows the multi-wavelength data with minimum and maximum temperature grey-body curves $(\beta=2)^{a}$ super-posed to illustrate the wide $(\sim 40 \mathrm{~K}-1000 \mathrm{~K})$ temperature range required in a pure dust emission scenario.

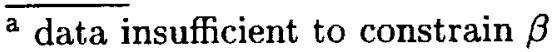

Smirnov test finds that the probability of the two samples being drawn from the same distributions are $<0.001(L(0.4-0.2 \mu \mathrm{m}) / L(0.8-0.4 \mu \mathrm{m}))$ and $<0.01$ $(L(0.4-0.2 \mu \mathrm{m}) / L(1.6-0.8 \mu \mathrm{m}))$. The remainder of the optical-infrared SED are remarkably similar between the two samples, supporting an interpretation in terms of reddening.

It is generally thought that the warm part of the infrared emission, $\lambda \lesssim 60 \mu \mathrm{m}$, is heated by the AGN while the cooler part is powered by starburst (STB) emission. Thus warm/cool infrared colors (e.g., $25 \mu \mathrm{m} / 60 \mu \mathrm{m}$ : Barthel, these proceedings; Heisler \& De Robertis 1999) give an indication of the relative importance of AGN and STB in a given source. Here we determine the luminosities of the AGN in the broad bands 3-60 $\mu \mathrm{m}$ and $60-200 \mu \mathrm{m}$ (Table 1) as an indication of the STB- and AGB-powered emission respectively. The ratios of these two luminosities are compared with the results of Polletta et al. (2000, and these proceedings) for a small AGN sample including both radioloud and radio-quiet sources (Figure 5). The HEAO AGN are confined to the cooler range a difference at $>95 \%$ significance (Kolmogorov-Smirnov, onetailed test). This is likely due to the presence of higher luminosity sources in the Polletta sample for which the cool dust (STB) contribution from their host galaxy is relatively weaker.

\section{High-redshift AGN}

Our ISO sample includes $8 \mathrm{AGN}$ in the redshift range $1.0 \leq z \leq 4.7$, several of which have existing/new SCUBA (Submillimeter Common-User Bolometer Array) observations from the JCMT (James Clerk Maxwell Telescope). As discussed in Wilkes et al. (2000), the SED of the high redshift sources vary 

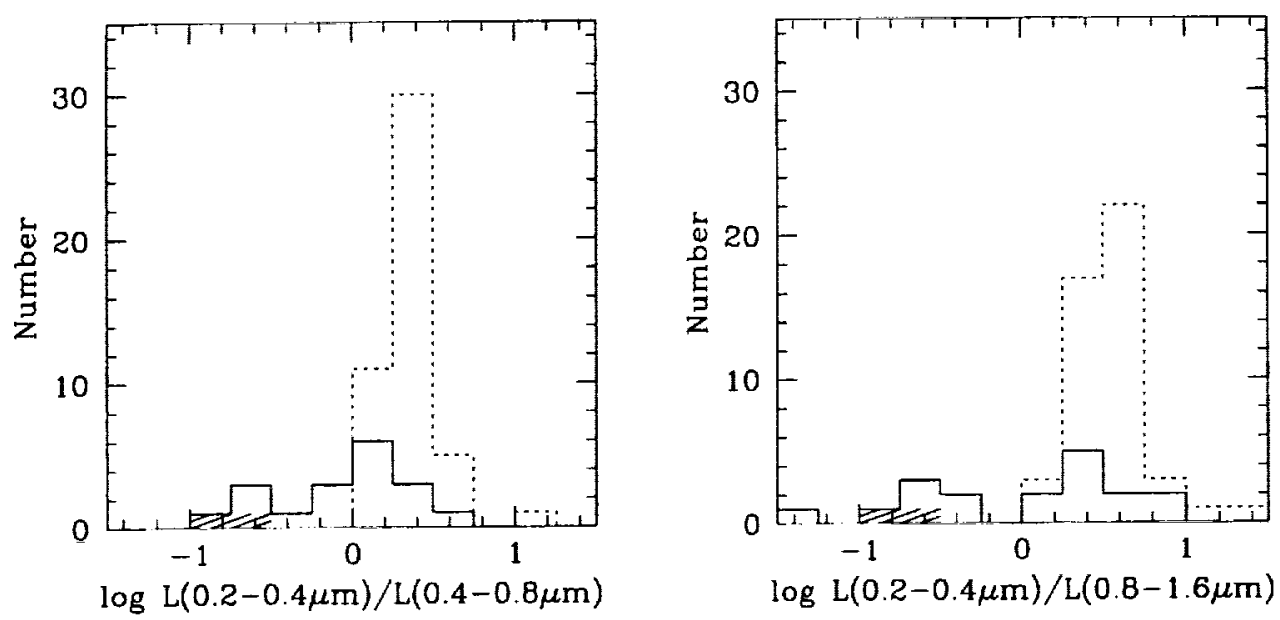

Fig. 4. The distribution of UV to optical (left) and UV to red (right) luminosity ratios for our X-ray selected AGNs (solid line) compared with those of the Elvis et al. (1994 - dotted line) sample, illustrating the extension of the X-ray sample to redder optical colors. Hatching indicates sources with significant intrinsic X-ray absorption.

widely so that, given the small sample, it is not possible to draw general conclusions for the high-redshift population. Figure 6 shows the current SED for $1508+5714$, including new submm data from SCUBA on the JCMT taken 3, 4 July 1999. Comparison with the low-redshift median SED (Elvis et al. 1994) shows that the ISO detections are $\sim 100$ times brighter than those expected based on the rest-frame optical fluxes to which the median is normalized.

\section{$4.1 \quad$ BR1202-0727}

This source was, for a while, thought to have the highest intrinsic near-infrared luminosity of any source in the universe based on its ISOPHOT fluxes (Wilkes et al. 1999, 2000). However, a comparison of the ISOPHOT fluxes observed at $7,12,25 \mu \mathrm{m}$ with the ISOCAM $8-15 \mu \mathrm{m}$ flux observed on 14 July 1996 , 5 days before our observation (Leech et al., these proceedings) shows a $\times 100$ discrepancy in flux levels. The source is very close to the ecliptic plane $\left(\beta \sim 6.5^{\circ}\right)$ and it seems likely that the detections are due to passage of an asteroid through the ISOPHOT beam rather than from the quasar. Possible candidates are currently being investigated. 


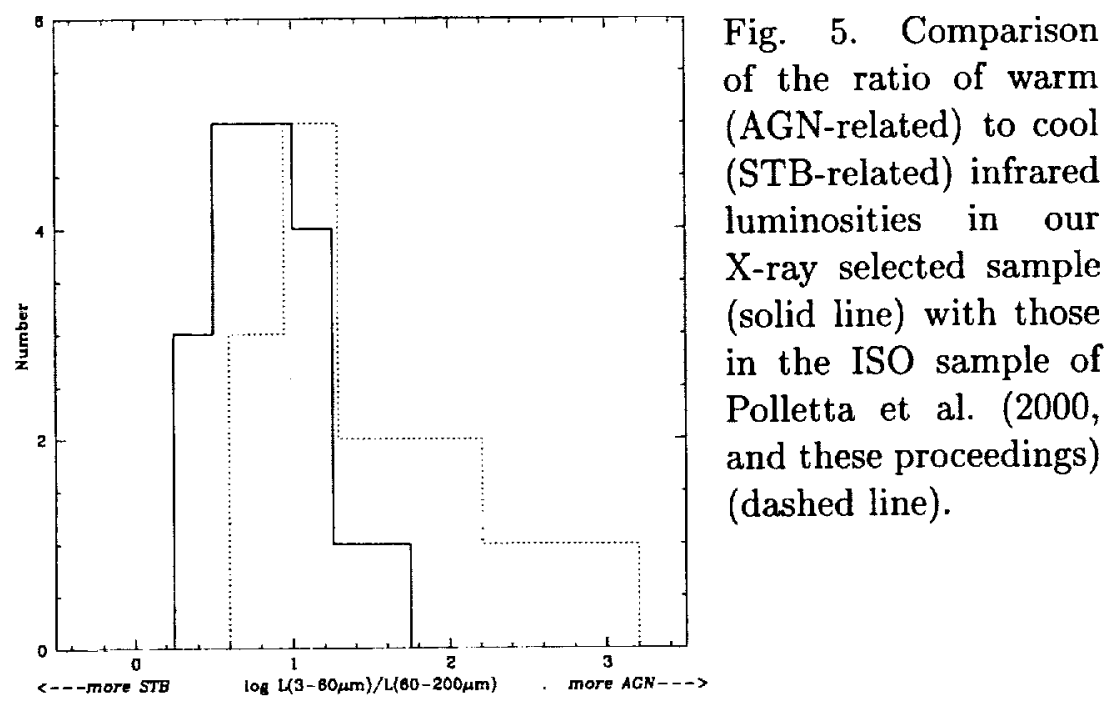

\subsection{Estimating the star-formation rate in high-redshift AGN}

The high far-infrared luminosities of a number of high-redshift quasars lead to estimates for dust masses $\sim 10^{8-9} \mathrm{M}_{\odot}$ (Hughes et al. 1997, Andreani et al. 1999), about 2 orders of magnitude larger than the Milky Way's current dust supply (Davies et al. 1997). Such large dust masses in high redshift quasars imply strikingly vigorous star-formation in the early universe given the little time available. The observations allow us to place limits on the star-formation rate (SFR) and to explore the question of whether star-formation or an AGN powers the far-infrared emission in these sources.

Using the far-infrared luminosities of 7 (rest-frame) far-infrared detected highredshift quasars in a sample of 35 drawn from our ISO sample and from the literature, we determine the dust mass based on a typical temperature of $50 \mathrm{~K}$. Assuming that the overall dust production efficiency is of order $1 \%$ (Blain et al. 1999), we estimate the mean SFR over the short interval between the epoch of initial star-formation, taken to be $z_{*}=10$, and the redshift of the quasar. The far-infrared luminosities implied by this mean SFR (10-50 $\left.\mathrm{M}_{\odot} \mathrm{yr}^{-1}\right)$ are typically about an order-of-magnitude below those observed $\left(\sim 10^{13} \mathrm{~L}_{\odot}\right)$, adopting $L_{\mathrm{FIR}}=1 \times 10^{10} \mathrm{SFR} \mathrm{M}_{\odot} \mathrm{yr}^{-1} \mathrm{~L}_{\odot}$ (Kennicutt 1998, Scoville \& Young 1983). Possible explanations for this discrepancy include an active nucleus powering $\sim 90 \%$ of the far-infrared luminosity or observation of these quasars during a powerful burst of star formation, $\geq 1000 \mathrm{M}_{\odot} \times \mathrm{yr}^{-1}$, about 10 times the rate seen in the brightest, local starburst galaxies such as Arp 220 (Anantharamaiah et al. 2000, Smith et al. 1998).

In the latter case, we can estimate the star-formation duty cycle at high redshift if we assume only those quasars in which a strong starburst is occurring 


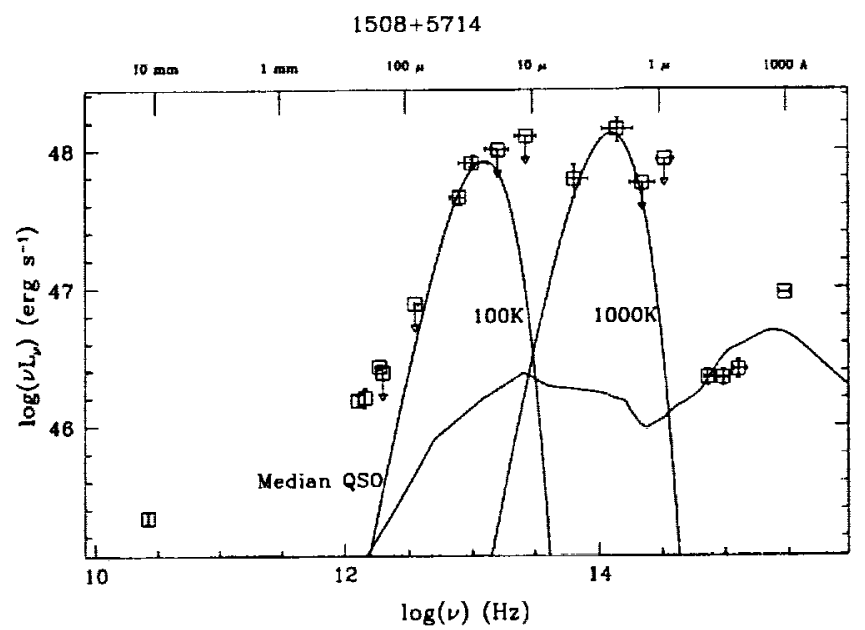

Fig. 6. The radio-UV SED of the high redshift quasar $1508+5714$. For comparison the median SED for low-redshift quasars (Elvis et al. 1994) is shown along with representative grey-body curves assuming $\beta=2$ illustrating the wide range of temperatures required in a purely thermal emission model.

are sufficiently bright to be detected in the far-infrared. Our sample of 35 sources includes 7 detections leading to an estimated duty cycle of 0.2 . The discrepancy is then reduced to a factor of $\sim 2$ with a corresponding decrease in the AGN-powered infrared luminosity. The uncertainties are too large to draw any strong conclusions. However, it is clear that either star formation at high-redshift occurs in relatively short, high rate bursts or the active nucleus is a significant contributor to the far-infrared luminosity in high-redshift quasars.

\section{Acknowledgements}

Heartfelt thanks are due to Dr. Eric Hooper, Dr. Joanna Kuraszkiewicz and Professor Kim McLeod for their hard work and support on the ISO Key Project which greatly contributed to this paper. I thank also my long-term collaborators: Martin Elvis, Chris Impey, Carol Lonsdale, Matt Malkan and Jonathan McDowell. The financial support of NASA grant NAG5-8847 (ISO) is gratefully acknowledged.

\section{References}

[1] Anantharamaiah K.R., Viallefond F., Mohan N.R., Goss W.M., Zhao J.H., 2000, ApJ 537, 613

[2] Antonucci R.R.J., Barvainis R., Alloin D., 1990, ApJ 353, 416

[3] Andreani P., Franceschini A., Granato G., 1999, MNRAS 306, 161

[4] Barvainis R., 1990, ApJ 353, 419

[5] Blain A.W., Smail I., Ivison R.J., Kneib J.-P., 1999, MNRAS 302, 632

[6] Brissenden, R., 1989, PhD Thesis, The Australian National University

[7] Carilli C.L., et al., 2000, ApJ 533, L13 
[8] Carleton N.P, Elvis M., Fabbiano G., et al., 1987, ApJ 318, 595

[9] Chini R., Kreysa E., Biermann, P.L., 1989, A\&A 219, 87

[10] Courvoisier T.J.-L., 1998, A\&AR 9, 1

[11] Davies J.I., Trewhella M., Jones H., et al., 1997, MNRAS 288, 679

[12] Efstathiou A., Rowan-Robinson M., 1995, MNRAS 273, 649

[13] Elvis M.S., Wilkes B.J., McDowell J.C., et al., 1994, ApJS 95, 1

[14] Gear W., et al., 1994, MNRAS 267, 167

[15] Granato G.L., Danese L., 1994, MNRAS 268, 235

[16] Granato G.L., Danese L., Franceschini A., 1997, ApJ 486, 147

[17] Green P.J., Anderson S.F., Ward M.J., 1992, MNRAS 254, 30

[18] Haas M., Chini R., Meisenheimer K., et al., 1998, ApJ 503, L109

[19] Haas M., et al., 2000, A\&A 354, 453

[20] Heisler C.A., De Robertis M.M., 1999, AJ 118, 2038

[21] Hoekstra H., Barthel P.D., Hes R., 1997, A\&A 319, 757

[22] Hooper E.J., Wilkes B.J., McLeod K.K., et al., 1999a, in The Universe as Seen by ISO, ESA SP-427, ed. P. Cox, \& M. Kessler (Paris: ESA), 893

[23] Hooper E.J., Wilkes B.J., McLeod K.K., et al., 1999b, in Astrophysics with Infrared Surveys: A Prelude to SIRTF, ASP Conf. Ser. 177, ed. M.D. Bicay, C.A. Beichman, R.M. Cutri, B.F. Madore, 153

[24] Hughes D.H., Dunlop J.S., Rawlings S., 1997, MNRAS 289, 766

[25] Hughes D.H., Robson E.I., Dunlop J.S., Gear W.K., 1993, MNRAS 263, 607

[26] Ivison R.J., Smail I., Le Borgne J.-F., et al., 1998, MNRAS 298, 583

[27] Kennicutt R.C., 1998, ApJ 498, 541

[28] Kessler M.F., 1999, in The Universe as Seen by ISO, ESA SP-427, ed. P. Cox, M. Kessler (Paris: ESA), 23

[29] Krabbe A., Böker T., Maiolino R., 2001, ApJ submitted, (astro-ph/0010119)

[30] Neugebauer G., Matthews K., 1999, AJ 118, 35

[31] Pérez-García A.M., Rodríguez-Espinosa J.M., Santolaya-Rey A.E., 1998, ApJ 500,685

[32] Pier E., Krolik J., 1992, ApJ 410, 99

[33] Polletta M., Courvoisier T.J-L., Hooper E.J., Wilkes B.J., 2000, A\&A 362, 75

[34] Remillard R., et al., 1986, ApJ 301742

[35] Sanders D.B., et al., 1989, ApJ 347, 29

[36] Schaerer D., Guseva N.G., Izotov Yu.I., Thuan T.X., 2000, A\&A 362, 53

[37] Scoville N.Z., Young J.S., 1983, ApJ 265, 148

[38] Smith H.F., Lonsdale C.J., Lonsdale C.J., Diamond P.J., 1998, ApJ 493, L17

[39] Tansley D., Birkinshaw M., Worrall D.M., 2001, in preparation

[40] Tuerler M., et al., 1999 A\&AS 134, 89

[41] Van Bemmel I.M., Barthel P.D., De Graauw, M.W.M., 2000, A\&A 359, 523

[42] Wilkes B.J., Hooper E.J., McLeod K.K., et al., 1999, in The Universe as Seen by ISO, ESA SP-427, ed. P. Cox, M. Kessler (Paris: ESA), 845

[43] Wilkes B.J., Hooper E.J., McLeod K.K., et al., 2000, in ISO Surveys of a Dusty Universe, Lecture Notes in Physics, 548, ed. D. Lemke, M. Stickel, K. Wilke, 177

[44] Wood K.S., et al., 1984, ApJS 56, 507 
